\title{
Noninvasive mechanical ventilation in high-risk pulmonary infections: a clinical review
}

\author{
Antonio M. Esquinas ${ }^{1}$, S. Egbert Pravinkumar², Raffaele Scala ${ }^{3}$, \\ Peter Gay ${ }^{4}$, Arie Soroksky ${ }^{5}$, Christophe Girault ${ }^{6}$, Fang Han ${ }^{7}$, \\ David S. Hui ${ }^{8}$, Peter J. Papadakos ${ }^{9}$ and Nicolino Ambrosino ${ }^{10}$ \\ on behalf of the International NIV Network ${ }^{11}$
}

\begin{abstract}
Affiliations: 'Intensive Care Unit, Hospital Morales Meseguer, Murcia, Spain. ${ }^{2}$ Dept of Critical Care, The University of Texas M.D. Anderson Cancer Center, Houston, TX, USA. ${ }^{3}$ Pulmonary and Respiratory Intensive Care Unit, S. Donato Hospital, Arezzo, Italy. ${ }^{4}$ Dept of Pulmonary, Critical Care and Sleep Medicine, Mayo Clinic, Rochester, MN, USA. ${ }^{5}$ General Intensive Care Unit, Assaf Harofeh Medical Center, Ramat Aviv, Israel. ${ }^{6}$ Dept of Medical Intensive Care, Charles Nicolle Rouen University Hospital, UPRES EA 3830-IRIB, Institute for Biomedical Research, Rouen University, Rouen, France. 'Dept of Pulmonary Medicine, The People's Hospital, Beijing University, Beijing, China. ${ }^{8}$ Dept of Medicine and Therapeutics, The Chinese University of Hong Kong, Hong Kong, SAR, China. ${ }^{9}$ Critical Care, University of Rochester, Rochester NY, USA. ${ }^{10}$ Pulmonary and Respiratory Intensive Care Unit, Cardio-Thoracic Dept, University Hospital Pisa, Pisa, Italy. ${ }^{11}$ For a full list of the International NIV Network collaborators see the Acknowledgements.
\end{abstract}

Correspondence: Antonio M. Esquinas, Intensive Care Unit, Hospital Morales Meseguer, Avenida Marques de Los Velez, s/n, Murcia, 3088-Spain. E-mail: antmesquinas@igmail.com

ABSTRACT The aim of this article was to review the role of noninvasive ventilation (NIV) in acute pulmonary infectious diseases, such as severe acute respiratory syndrome (SARS), H1N1 and tuberculosis, and to assess the risk of disease transmission with the use of NIV from patients to healthcare workers.

We performed a clinical review by searching Medline and EMBASE. These databases were searched for articles on "clinical trials" and "randomised controlled trials". The keywords selected were non-invasive ventilation pulmonary infections, influenza-A (H1N1), SARS and tuberculosis. These terms were crossreferenced with the following keywords: health care workers, airborne infections, complications, intensive care unit and pandemic. The members of the International NIV Network examined the major results regarding NIV applications and SARS, H1N1 and tuberculosis. Cross-referencing mechanical ventilation with SARS yielded 76 studies, of which 10 studies involved the use of NIV and five were ultimately selected for inclusion in this review. Cross-referencing with H1N1 yielded 275 studies, of which 27 involved NIV. Of these, 22 were selected for review. Cross-referencing with tuberculosis yielded 285 studies, of which 15 involved NIV and from these seven were selected. In total 34 studies were selected for this review.

NIV, when applied early in selected patients with SARS, H1N1 and acute pulmonary tuberculosis infections, can reverse respiratory failure. There are only a few reports of infectious disease transmission among healthcare workers.

@ERSpublications

NIV in high risk pulmonary infection management can prevent respiratory failure in ICUs with well trained staff http://ow.ly/wSpqx

Received: Dec 212013 | Accepted after revision: May 112014

Conflict of interest: None declared.

Provenance: Submitted article, peer reviewed.

The present work was previously presented as a poster presentation at the American Thoracic Society 2010 Interational Conference (New Orleans, LA, USA).

Copyright OERS 2014. ERR articles are open access and distributed under the terms of the Creative Commons Attribution Non-Commercial Licence 4.0. 


\section{Introduction}

Noninvasive ventilation (NIV) as an early option in selected contagious patients with severe acute respiratory syndrome (SARS), H1N1 and tuberculosis (TB) infections that can avert or reverse respiratory failure and, therefore, decrease complications when implemented in units with well-trained staff. Currently, there are only a few reports of infectious disease transmission among healthcare workers. Practical precautions and protocols to protect healthcare workers during NIV use in high-risk pulmonary infections are necessary.

NIV is an essential component in both emergency and intensive care medicine [1]. In the 1950s, the widespread use of the iron lung during the polio epidemic increased the survival of patients with respiratory failure [2,3]. Although the use of NIV and the number of articles on NIV have been rapidly growing over the past decades [3-5], there are still insufficient data concerning the use of NIV during pulmonary infections, especially in pandemic respiratory infections. NIV was used in patients with SARS in 2002-2003 and also during the H1N1 epidemic in 2009. However, in recent years NIV use has been extended to patients with respiratory failure due to a wide spectrum of infectious diseases. These include, but are not limited to, SARS and pandemic avian influenza (H5N1). However, some concern has been raised with the use of NIV in patients with contagious diseases. Studies from Mexico, Canada, Spain, and Australia have reported experiences with treating $\mathrm{H} 1 \mathrm{~N} 1$ influenza patients with respiratory failure [5, 6]. A significant proportion of these patients were treated with NIV. There were no reports of disease transmission with the use of NIV from patients to healthcare workers in these studies, but all involved healthcare workers were not routinely screened for infection. Nevertheless, the World Health Organization (WHO) has included NIV among aerosol-generating procedures in which the risk of pathogen transmission is possible $[7,8]$.

The aim of our study was to conduct a systematic review to determine the effectiveness of NIV in high-risk pulmonary infections, including pandemic infections, and to explore the risk of infectious spread among healthcare workers with the use of NIV. In order to perform this review, we formed an international working group comprised of experts in infectious pathology and NIV from Asia, Europe, Latin America and the USA. This network is a permanent working group that evaluates technological implications concerning NIV for future pandemics and mass disaster causalities. We selected key topics related to pertinent methodological and technical issues in NIV and infectious diseases. Our study centred on the use of NIV in pulmonary infections, especially during pandemics, to determine the risk of transmission of infection to healthcare workers. The international members of this network participated in the careful review of selected studies in order to provide recommendations for protocols and guidelines, and subsequently to the writing of this manuscript.

We conducted a search in Medline (PubMed) for published randomised and non-randomised control trials, as well as observational studies (cohort, case-control or case series) in adult patients between January 1990 and March 2012, and in EMBASE from 1966 to June 2012.

The keywords selected were: non-invasive ventilation, pulmonary infections, influenza A (H1N1), SARS and tuberculosis. These terms were cross-referenced with the following keywords: health care workers, airborne infections, complications, intensive care unit and pandemic. Bibliographies of the selected articles were hand searched for additional relevant articles. We did not include paediatric studies.

\section{Effectiveness of NIV in pulmonary infections and pandemics} NIV in SARS

SARS, caused by the novel coronavirus (SARS-CoV), emerged in November 2002 and was first identified by the WHO in mid-March 2003. SARS is a severe and progressive disease with $\sim 25 \%$ of patients developing respiratory failure [9].

In order to reduce endotracheal intubation (ETI) and its associated complications, NIV was commonly employed in the SARS outbreak in China, including Hong Kong. The use of NIV has become part of the standard treatment protocol for SARS [10]. A summary of clinical studies and corresponding recommendations are described in table 1.

NIV does not demonstrate absolute benefit among non-chronic obstructive pulmonary disease (COPD) patients with acute hypoxaemic respiratory failure or acute respiratory distress syndrome (ARDS), e.g. in SARS patients $[8,9,11-17]$. It is well known that ETI is associated with higher risk of disease transmission and associated complications. The use of NIV as initial ventilator support for respiratory failure in the presence of SARS appears to be a reasonable option, albeit under strict infection control measures. 


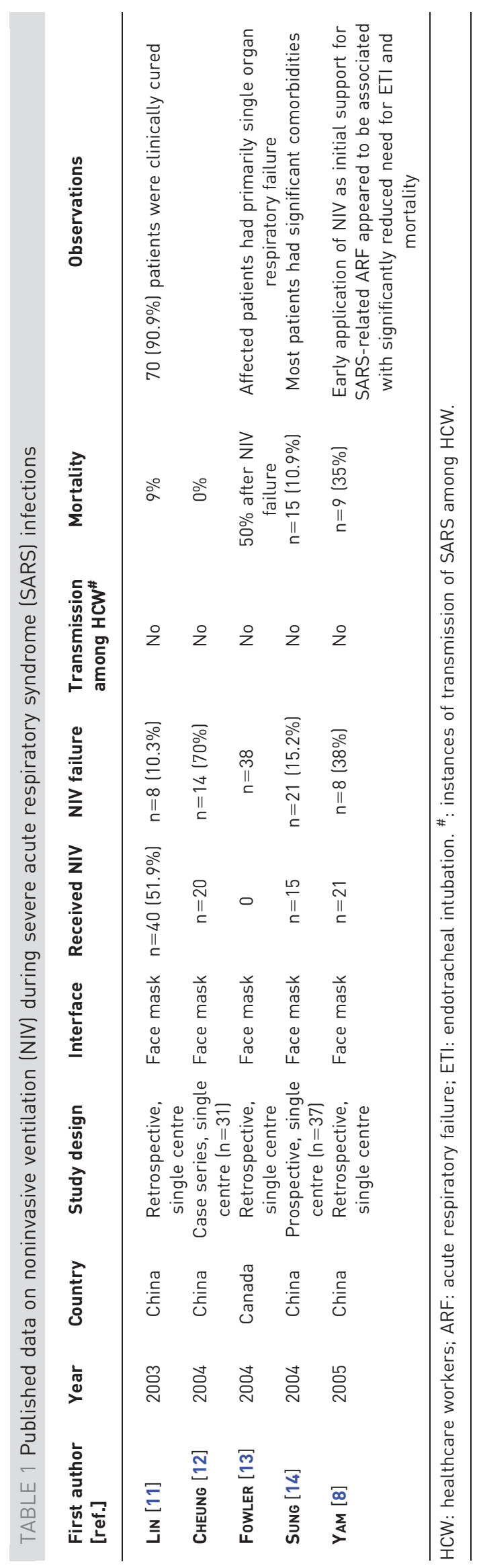




\section{NIV in influenza A H1N1 infection}

Pandemic influenza A (pH1N1) is a new strain of influenza virus that was first identified in Mexico and the USA in the early part of 2009 [18-30]. The pH1N1 virus originated from the swine influenza (H1N1) virus circulating in North American pigs [23].

Animal studies have shown that the novel influenza virus caused increased morbidity and replicated to higher titers in lung tissue; thus, explaining its pathogenicity and ability to invade the lower respiratory tract in humans, resulting in rapid and fulminant respiratory failure [19]. Such clinical deterioration is characterised by sudden and rapidly progressive respiratory failure with persistent and refractory hypoxia, bilateral diffuse pulmonary infiltrates and a low arterial oxygen tension $\left(\mathrm{PaO}_{2}\right)$ /inspiratory oxygen fraction $\left(\mathrm{FIO}_{2}\right)$ ratio reaching ARDS criteria [20-22, 25, 26, 31, 32]. Severe respiratory failure is common during the first week and the incidence decreases over time. Refractory hypoxia was the major cause of death, followed by multi-organ failure and shock [26-28, 32].

About $10-30 \%$ of hospitalised patients required admission to the intensive care unit (ICU) during the pH1N1 epidemic $[27,28,32]$. Comorbidities were noted in $32-84 \%$ of cases admitted to the ICU. These included obesity, COPD, diabetes mellitus, asthma, immunosuppression, chronic kidney disease and heart failure [27-30, 32]. The overall ICU mortality rate for critically ill cases of pH1N1 was close to 17\% [19-33]. Factors that were independently associated with mortality included requirement for invasive mechanical ventilation (IMV) and a low $\mathrm{PaO}_{2} / \mathrm{FIO}_{2}$ ratio at ICU admission, the presence of comorbidities and older age $[19,20,22,23]$. Autopsy findings showed three distinct pulmonary pathologies: diffuse alveolar damage, necrotising bronchiolitis, and diffuse alveolar damage with alveolar haemorrhage [21, 23, 24, 31]. If we analyse ventilatory management, IMV with lung protective ventilatory strategy and fluid restriction is recommended as the initial approach for managing patients with $\mathrm{pH} 1 \mathrm{~N} 1$ infection complicated by ARDS $[21,24,26,27,32,33]$.

Based on the guidelines from the European Respiratory Society (ERS)/European Society of Intensive Care Medicine (ESICM), WHO, the UK National Health Service, Hong Kong Lung Foundation and the American Association for Respiratory Care (AARC), NIV is not to be used as first-line therapy in pH1N1associated respiratory failure for the following reasons. 1) Poor clinical efficacy in severe respiratory failure that rapidly progresses to refractory hypoxaemia and ARDS. 2) The prevalent pattern of hypoxaemic failure instead of hypercapnic respiratory failure in patients with pH1N1. 3) Concern about aerosol droplet particle dispersion and spread of infection [10, 19, 34, 35].

A summary of published studies on NIV in pH1N1 is shown in table 2 [19-33, 36-48]. Of the 22 studies included in table 2, the majority were case series reports. The contribution to $\mathrm{pH} 1 \mathrm{~N} 1$ case series according to country is shown in table 3. There is only one international study [37] that has shown a favourable result with the use of NIV in pH1N1. Five of the studies, most of which were case series applying a face mask for NIV, yielded a failure rate of $10-15 \%$. A full face mask was the NIV interface choice in all but one study; in which the NIV helmet and face mask were used as an alternative. The results regarding the success of NIV were highly variable. Among the causes of NIV failure are refractory hypoxaemia and shock, with multiorgan failure being the most unfavourable. As shown in table 2, mortality is clearly lower in the group treated with NIV versus the IMV group. While the Chinese and European studies appear to argue in favour of NIV use, the Canadian study did not support its use. These studies do not report on significant complications for patients and healthcare workers and in none of these studies do the authors report cases of infection transmission to healthcare workers.

Based on the review of these reports our conclusions are as follows. NIV has a role in the management of early respiratory failure due to $\mathrm{pH} 1 \mathrm{~N} 1$ infection; however, in a strictly controlled environment with close monitoring of healthcare workers and with adequate precautions for prevention of infection transmission [20, 22, 24-26, 29-33, 36-48]. NIV has no role in severe respiratory failure and ARDS related to severe pH1N1 infection. These patients must undergo ETI and IMV [26, 30, 32, 33, 42-44, 46].

\section{NIV in pulmonary TB}

NIV remains an option for ventilatory support in both acute and chronic respiratory failure secondary to pulmonary TB [49-54]. In acute situations, judicious use of NIV in carefully selected patients can potentially obviate the need for IMV [49-53, 55-58]. NIV can be regarded as a simple and suitable solution not only for the provision of long-term and domiciliary-assisted ventilation in patients with sequelae of pulmonary TB, but also for acute exacerbations of pulmonary $\mathrm{TB}[50,54,59,60]$. The concern with the use of NIV in such acute situations is linked with the potential risk of the transmission of TB [49, 56]. Whether or not NIV should be considered a high-risk procedure in infectious diseases like TB continues to be a controversial issue $[49,56]$. However, the limited availability of invasive ventilators and/or ICU beds, 


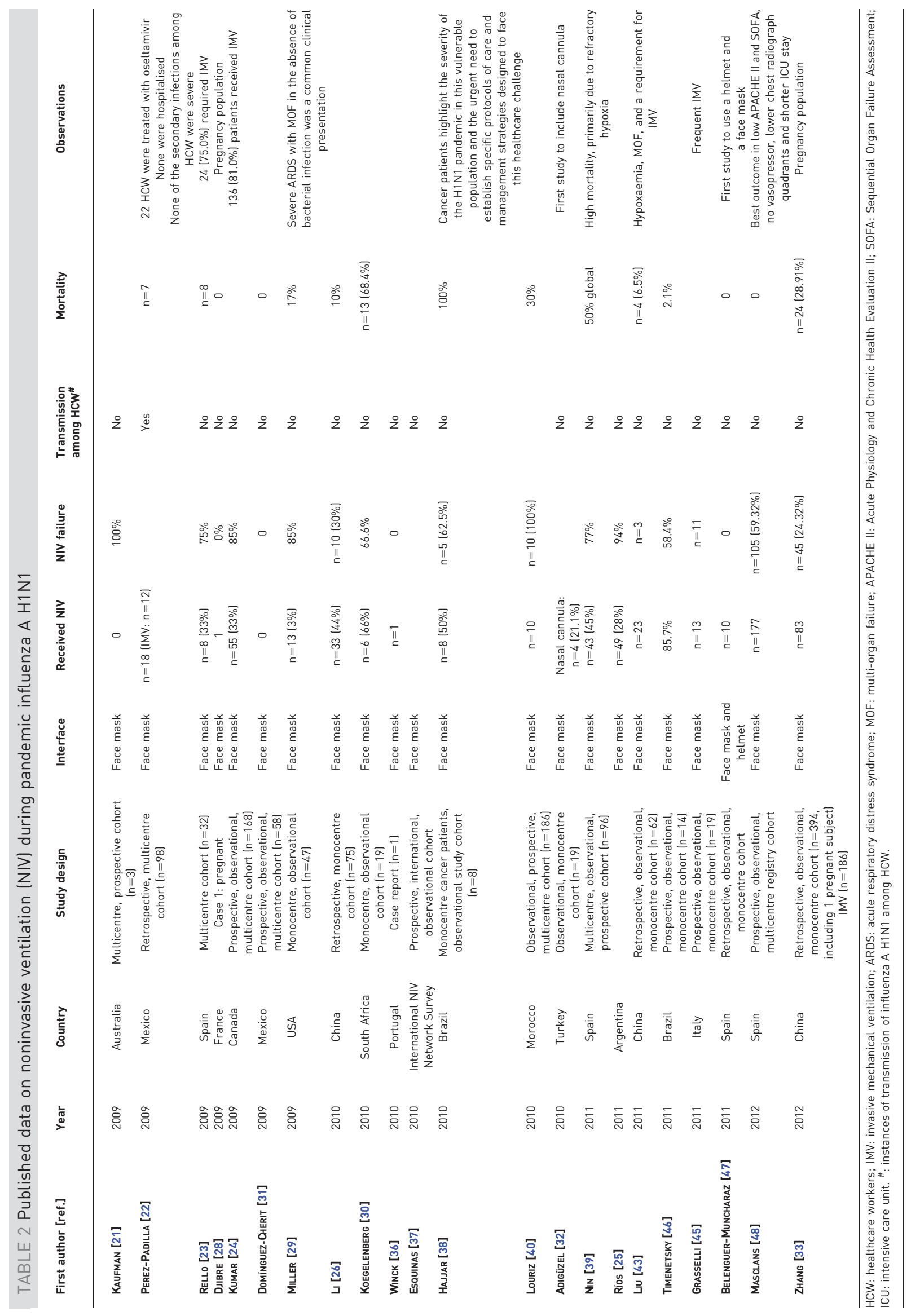


TABLE 3 Contribution to pandemic influenza A H1N1 case series (total $n=23$ ) based on country

$\begin{array}{lr}\text { European countries } & \mathrm{n}=9 \\ \text { Spain } & \mathrm{n}=5 \\ \text { Italy } & \mathrm{n}=1 \\ \text { France } & \mathrm{n}=1 \\ \text { Portugal } & \mathrm{n}=1 \\ \text { Turkey } & \mathrm{n}=1 \\ \text { Asia } & \mathrm{n}=3 \\ \text { North America } & \mathrm{n}=1 \\ \text { Australia } & \mathrm{n}=1 \\ \text { Canada } & \mathrm{n}=1 \\ \text { Latin America } & \mathrm{n}=6 \\ \text { Mexico } & \mathrm{n}=2 \\ \text { Brazil } & \mathrm{n}=2 \\ \text { Chilean-Uruguay } & \mathrm{n}=1 \\ \text { Argentina } & \mathrm{n}=1 \\ \text { South Africa } & \mathrm{n}=1 \\ \text { North Africa (Morocco) } & \mathrm{n}=1\end{array}$

especially in developing countries where TB incidence remains high, is an important constraint in managing patients with TB and acute respiratory failure by means of NIV.

The medical literature offers very few published reports concerning the use of NIV in contagious TB patients. This is due to the fact that there is a limited number of published articles where NIV was used in exacerbations of chronic respiratory failure in patients with TB sequelae [50, 54, 59, 60], and even fewer reports were available about the use of mechanical ventilation in acute respiratory failure due to active pulmonary TB $[56,57]$.

In the seven published studies dealing with patients suffering acute exacerbations of pulmonary TB sequelae, nasal mask was the most frequently used interface for delivery of NIV (used in four studies). These studies suggest no apparent complications for patients and workers and report no cases of death in treated patients. Continued applications at home following the acute phase yielded similar results. A summary of published data on NIV in acute exacerbation of pulmonary TB is shown in table 4.

In summary, the practitioner who has to decide whether to initiate NIV or IMV in acute respiratory failure due to pulmonary TB has to take into account several factors, such as: the prolonged period of infectiousness of these patients; the lag time for noticeable clinical improvement following initiation of treatment; and the risks related to positive pressure ventilation, such as pneumothorax and haemoptysis [49].

\section{Risk of aerosol droplet generation and infection spread with NIV}

Cough represents the most important airway defence reflex and one of the main symptoms of respiratory diseases $[7,61,62]$. During coughing and sneezing, particles of mucus can be expelled to a distance of up to $9 \mathrm{~m}[14,13,62]$. Therefore, various pathogens, when present, could infect surrounding people and animals, thus markedly contributing to the massive dissemination of airborne infections [62]. It was shown that, during a 3 -h flight, $\sim 80 \%$ of passengers could potentially become infected by a coughing patient with influenza. Subsequently, these passengers could disseminate the infection at their destinations worldwide $[14,63]$. Urgent protective and therapeutic actions are considered necessary for $\mathrm{pH} 1 \mathrm{~N} 1$, SARS and TB, which mostly affect immune-compromised populations [8, 12, 16, 47].

Currently, available recommendations are mainly based on published studies and experiences from the first SARS epidemic in 2003 [8, 11-17]. The pivotal study arguing that NIV poses a high risk for spread of infection is based on the assessment of particle dispersion using an experimental model. Smoke was introduced into the lungs of a mannequin while NIV was being performed. Plumes of smoke emerging from the vented mask were photographed for particle dispersion. As yet, there have been no studies evaluating particle dispersion in human beings. Much debate has risen since the SARS epidemic as to whether a mannequin-based study on particle dispersion simulates a real patient using NIV. Many experts argue that the NIV mask may in fact offer protection from secretions that would otherwise disperse from the infected patient during coughing, sneezing and speaking. Furthermore, there is no controlled data comparing particle dispersion between individuals receiving NIV and those who did not $[9,62]$.

Guidelines from ERS/ESICM, WHO, the UK National Health Service, Hong Kong Lung Foundation and the AARC, have considered NIV as a high-risk procedure during respiratory pandemics. This has led to 


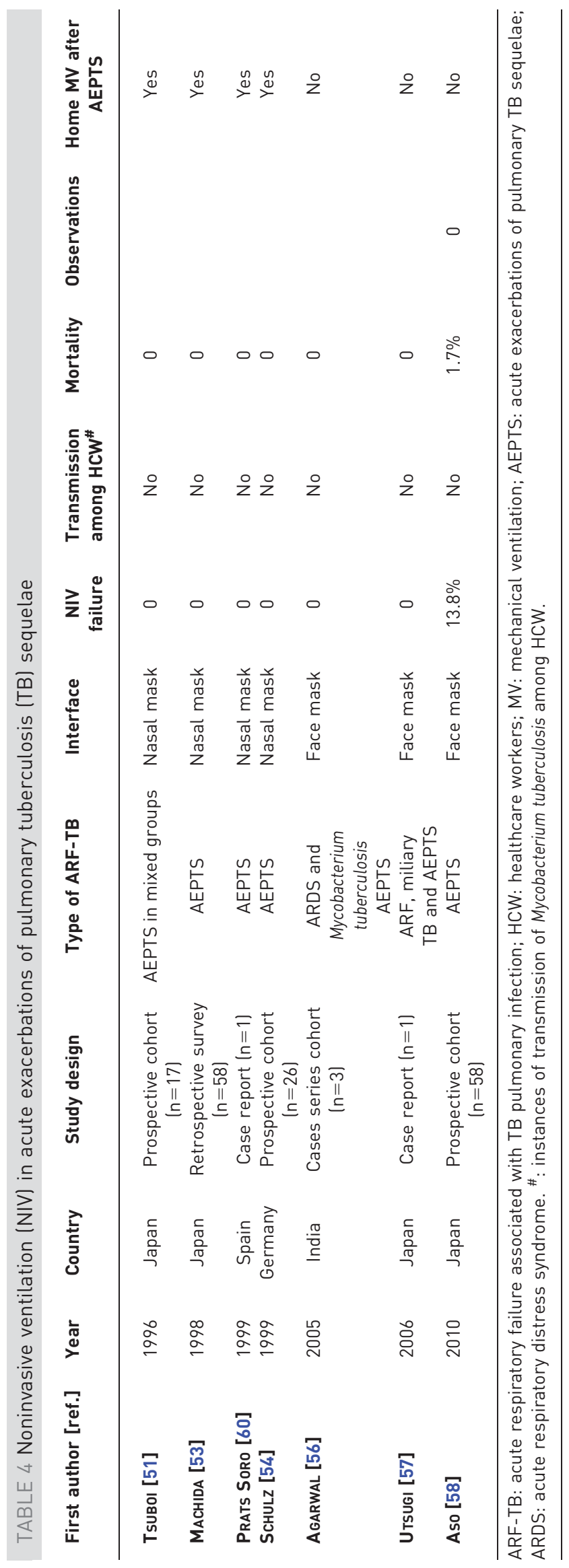


overuse of ICU beds and strain on available resources, in addition to an increase in IMV-related complications. Further validation of the association between NIV and infection spread by particle dispersion is needed for planning the best strategy for future pandemics $[5,10,19,34,35,47,63]$.

Technical issues should also be considered in the strategic planning of guidelines that could be used by NIV teams for the management of suspected or proven cases of acute respiratory failure caused by contagious diseases $[34,63]$. When considering the choice of ventilators, physicians should give preference to machines equipped with a dual-limb circuit without an unfiltered expiratory port (i.e. plateau exhalation valve, anti-rebreathing valve, etc.) $[7,13,18,34,36]$. Such technology avoids the dispersion of expired air contaminated by infected particles through a circuit leak. Such leaks are caused by bias flow necessary to prevent rebreathing in single-limb circuits and are magnified by the high positive end-expiratory pressure levels that are often required in the treatment of acute hypoxaemic respiratory failure [7].

A recent study reported that the use of certain standard face masks for NIV in patients with infectious diseases might be associated with a substantial exposure to infectious organisms in the exhaled air occurring within a distance of $1 \mathrm{~m} \mathrm{[7].} \mathrm{The} \mathrm{dispersion} \mathrm{of} \mathrm{infected} \mathrm{respiratory} \mathrm{droplets} \mathrm{may} \mathrm{be} \mathrm{amplified} \mathrm{by} \mathrm{an} \mathrm{increase}$ in mask leakage, and can be aggravated with higher inspiratory pressures [7, 62].

Regarding interface selection, full face or total face masks might be preferred to nasal masks in order to theoretically prevent the potential spread of contaminated exhaled air particles from unintended air leaks through the mouth. Therefore, choosing the brand and size of mask that best fits the anatomy of the patient's facial contours and at the same time allows delivery of adequate pressure levels is crucial to minimise unintended air leaks around the interface. However, currently there is no published data to confirm this theoretical strategy as having a positive impact in clinical situations $[7,62]$.

Recently, in Europe, a helmet interface has become available for delivering NIV [3]. In the majority of patients, a helmet is better tolerated than a face mask [64]. It is speculated that the use of a helmet interface, where available, for NIV together with negative pressure rooms equipped with high-efficiency particulate air filters, may decrease the dispersion of infected respiratory droplets.

\section{Risk of infection spread with NIV in pandemics}

During the SARS pandemic, nearly half of all SARS victims were healthcare workers, highlighting the occupational risks associated with the care of infectious pneumonias and pandemic infections [11-14]. Evidence related to airborne transmission of the SARS virus and the potential risk of aerosol generation with the use of NIV in SARS still remain controversial [8, 9, 11-17].

FOWLER et al. [13] examined transmission rates in healthcare workers caring for SARS patients who required ETI and IMV. This study showed a greater risk of developing SARS in physicians and nurses performing ETI (relative risk 13.29 (95\% CI 2.99-59.04); $\mathrm{p}=0.03$ ). Although nurses caring for infected SARS patients who are on NIV may have been at increased risk of droplet infections (RR 2.3 (95\% CI 0.25-21.76)), this theoretical health-related risk was not translated into statistically significant clinical events $(\mathrm{p}=0.5)$. Two other reports analysed cases of SARS in Hong Kong, China, and there were no reported cases of SARS among healthcare workers caring for patients treated by NIV. However, it is unclear how this surveillance was carried out $[8,12,17,34]$.

YU et al. [17] conducted a case-control study to investigate nosocomial spread of SARS by identifying "super spreading events" in multiple hospitals in China, including Hong Kong $[8,12,16,17]$. The results were of importance since $71.1 \%$ and $74.8 \%$ of the infections were attributable to super spreading events in Hong Kong and Singapore (Malaysia), respectively. In the final multiple-logistic model, the use of NIV was one of the six factors found to be associated with the risk of dissemination [20, 21]. However, the significance of NIV as a contributing factor to a rapidly spreading healthcare event could not be established $[8,12-14,15-17]$.

To date, there are no reports demonstrating an increased risk of NIV-related dissemination in healthcare workers treating infected patients [10]. During the SARS outbreak, a study performed in Hong Kong by CHeung et al. [12] examined the efficacy of NIV in early ARDS and also evaluated the infection risk among healthcare workers who had direct contact with patients on NIV. A total of 22 (25\%) patients needed NIV and a total of 155 healthcare workers, including doctors, nurses and healthcare assistants, were exposed to these patients on NIV therapy and were regularly screened for signs of infection. Coronavirus serology was obtained in $97 \%$ of healthcare workers. NIV equipped with expiratory bacterial and viral filters was provided in isolated cubicles in the ward or ICU, which were centrally air-conditioned, and fitted with exhaust ventilation fans to achieve negative pressure flow. This study concluded that NIV was not only effective in preventing IMV in $70 \%$ of patients with acute respiratory failure due to SARS but effectively reduced the ICU length of stay or avoided ICU admission. Moreover, no infection was noted in any of the 
155 healthcare workers. Their serology tests for coronavirus were negative. The potential risk of particle dispersion and spread of infection due to NIV must not be overlooked, even though, at present, the data remains inconclusive.

In pulmonary $\mathrm{TB}$, the majority of patients have a relatively long period of infectiousness (weeks to months), until the diagnosis is established and an effective anti-TB therapy is initiated [49]. There are certain characteristics in TB patients that increase the risk for infectiousness: presence of cough, cavitation on chest radiograph, sputum smear-positive for acid-fast bacilli, and failure to cover the mouth and/or nose during coughing or with cough-inducing and aerosol-generating procedures $[49,50,56,58]$.

Mycobacterium tuberculosis is carried in airborne particles as droplet nuclei that can be generated when persons with pulmonary or laryngeal TB cough, sneeze, shout or sing. The infective particles are $\sim 1-5 \mu \mathrm{m}$ in diameter and are maintained in the air and spread throughout the room/building by air currents. Therefore, TB infection is transmitted by air, not by surface contact. Contact with oculonasal secretions, saliva or other fluids from the patient do not represent a mode of transmission of M. tuberculosis [58, 60].

\section{High-risk infections and healthcare worker protocols}

The AARC recently issued a statement concerning ventilation in mass casualty situations, including pandemics [63]. The recommendation is against the use of NIV in pandemic influenza as the disease often progresses to ARDS, for which NIV is not the standard of care [63]. The development of infection control protocols for healthcare workers is essential to avoid the risk of acquiring contagious diseases $[10,18,19,34$, 35, 49, 63, 65]. Protocols should be established in advance, before the onset of a crisis. Protocol should

TABLE 5 Summary of recommendations for noninvasive ventilation (NIV) during severe acute respiratory syndrome (SARS), H1N1 and tuberculosis (TB) infections

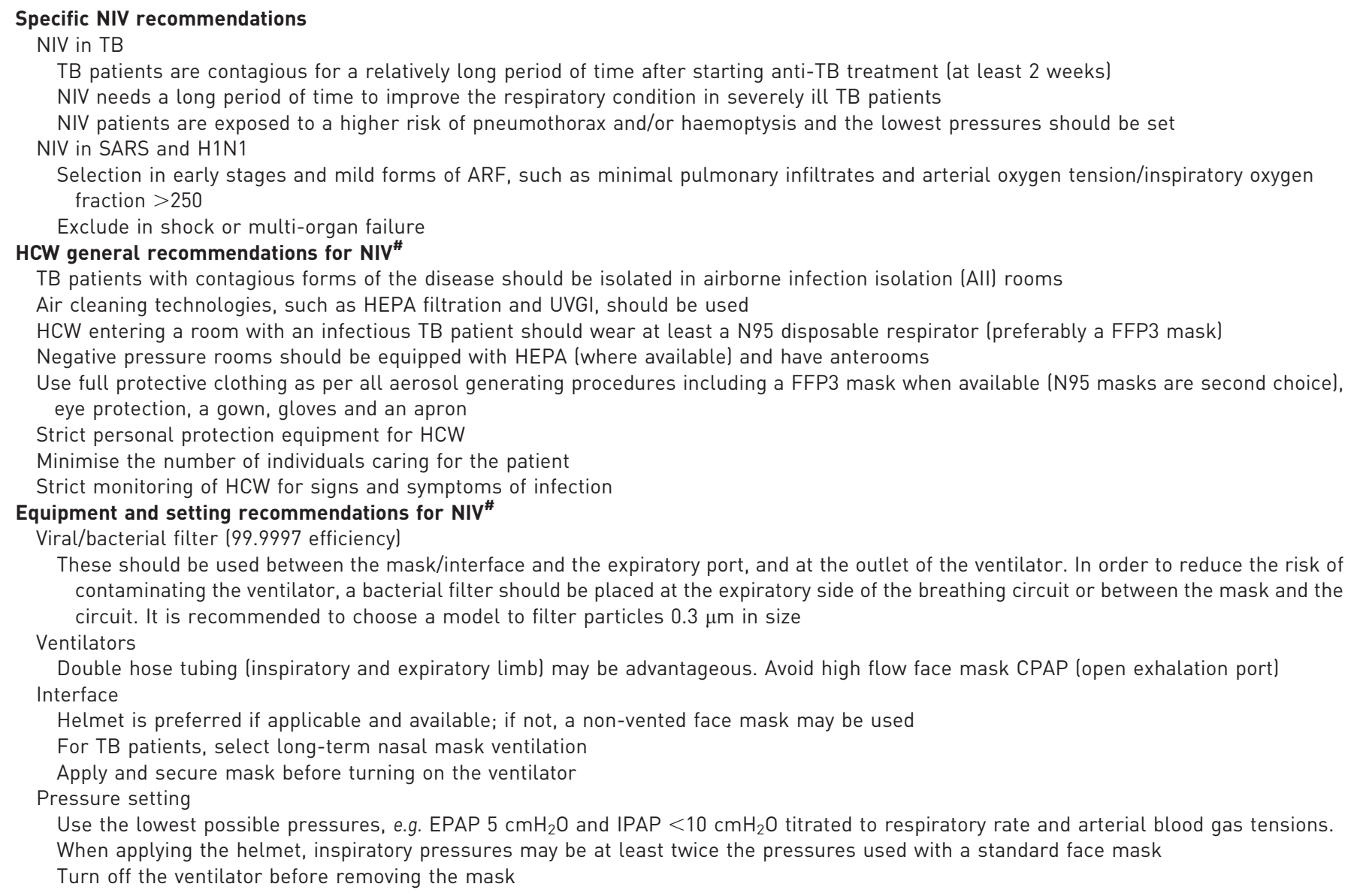

ARF: acute respiratory failure; HCW: healthcare workers; HEPA: high-efficiency particulate air; UVGI: ultraviolet germicidal irradiation; CPAP: continuous positive airway pressure; EPAP: expiratory positive airway pressure; IPAP: inspiratory positive airway pressure. ${ }^{\#}$ : these apply to SARS, H1N1 and TB. 
include handling procedures pertaining to aerosol-generating devices such as NIV, environment, equipment disinfection, and transport of infected patients. The designed protocol should be multidisciplinary in nature and involve doctors, nurses, physical therapists and technical personnel [10, 18, 19, 34, 35, 49, 63, 65]. However, these protocols are not intended to supplant physician judgment with respect to special clinical situations or individual patients. NIV must be managed under strict isolation measures in patients suspected of or diagnosed with highly contagious infections, with adequate protection of the healthcare workers who attend to the patient $[10,18,19,34,35,49,63,65]$.

Infected patients should be isolated in rooms with negative pressure, and visits should be restricted. The isolation time will depend on: the period of replication of the microorganism; comorbidity of the patient, such as immunosuppression; and availability of scientific information [10, 18, 19, 34, 35, 49, 63, 65]. Healthcare workers must wear personal protective equipment, as recommended by the Centers for Disease Control and Prevention's guidelines. These include N95 masks, gloves, gowns, caps, and face shields or goggles, complemented by strict hand hygiene before and after entering the room and after managing the patient $[10,18,19,34,35,49,63,65]$. It is essential that the healthcare workers are familiar with NIV and should have received adequate training for the use of NIV in contagious patients. They should also adhere strictly to the use of personal protective equipment to avoid the risk of acquiring contagious diseases. Recommendations for NIV in SARS, pH1N1 and TB are summarised in table 5.

\section{Conclusion}

NIV use in the management of acute respiratory failure in pulmonary infections, especially in pandemics, can avert or reverse respiratory failure and, therefore, decrease the rate of ETI in selected groups of contagious patients $[10,18,19,34,35,49,63,65]$. However, its use as first-line therapy is not recommended in severe pH1N1 infections, severe respiratory failure with ARDS or in pneumonia. Although only a few reports have been published to date on infectious disease transmission among healthcare workers who manage patients receiving NIV therapy, it is crucial that reasonable and adequate precautionary steps are followed in order to protect healthcare workers from an infectious spread, as well as other patients and family members. The International NIV Network recommends further research is conducted in this area to determine whether currently proposed NIV protocols are effective in reducing infectious particle dispersion and spread of disease to others.

\section{Acknowledgements}

The International NIV Network collaborators are as follows. Henry Kwok (Hospital Authority Head Office, Queen Elizabeth Hospital, University of Hong Kong, Hong Kong, China), Anca Macri (Clinica de Pneumologie U.M.F. "Carol Davila", Institutul de Pneumologie "Marius Nasta”, Bucharest, Romania), Ritesh Agarwal (Dept of Pulmonary Medicine, Postgraduate Institute of Medical Education and Research, Chandigarh, India), Benan Bayrakci (Dept of Pediatrics, School of Medicine, Hacettepe University Pediatric Intensive Care Unit, Ankara, Turkey), Dominic Dellweg (Dept of Respiratory and Critical Care Medicine, Kloster Grafschaft, Schmallenberg, Germany), Emilio Curiel (Intensive Care Unit, Hospital Carlos Haya, Malaga, Spain), Francesco Mojoli (Servizio di Anestesia e Rianimazione I, Fondazione IRCCS Policlinico S. Matteo, Pavia, Italy), Paolo Feltracco (Dept of Pharmacology and Anesthesiology, University Hospital of Padova, Padova, Italy), Praveen Neema (Dept of Anaesthesiology, Sree Chitra Tirunal Institute for Medical Sciences and Technology, Trivandrum, India), Stephan Steiner (Dept of Cardiology, Pneumology and Angiology, Heinrich Heine University Düsseldorf, Düsseldorf, Germany), Alexander Zarbock (Dept of Anesthesiology and Critical Care Medicine, University of Münster, Münster, Germany),Wei-Erh Cheng, (Division of Pulmonary and Critical Care Medicine, Dept of Internal Medicine, China Medical University Hospital, Taichung, Taiwan, China), Viliam Donic (Dept of Physiology and Sleep Laboratory, Faculty of Medicine, University of Pavol Jozef Safarik, Kosice, Slovakia), Pieter Depuydt (Dept of Intensive Care Medicine, Ghent University Hospital, Gent, Belgium), José Luis Sandoval Gutiérrez (Medicina InternaMedicina Crítica-Neumología, Departamento de Medicina Crítica y Terapia Intensiva, Centro de Investigación en Enfermedades Infecciosas, Instituto Nacional de Enfermedades Respiratorias (INER), Distrito Federal, Mexico), Yoshinori Matsuoka (Dept of Anesthesiology and Critical Care, Saga Medical School Hospital, Saga, Japan), Ana Paula Goncalves (Intensive Care Unit, Unidade Local de Saúde-Hospital, Guarda, Portugal), Belén Estébanez (Unidad de Cuidados Intensivos, Hospital del Tajo, Madrid, Spain), Güniz Koksal (Dept of Anaesthesiology and Reanimation, Istanbul University Cerrahpasa Medical Faculty, Istanbul, Turkey), Cenk Kirakli (Dept of Pulmonary and Critical Care, Intensive Care Unit, Dr. Suat Seren Chest Diseases and Thoracic Surgery Training and Research Hospital, Izmir, Turkey), Killen Harold Briones Claudett (Pulmonology Dept, Military Hospital, Guayaquil, Ecuador), Aurelio Rodriguez Fernandez (Unidad de Medicina Intensiva y Emergencias, Hospital "Saturnino Lora", Santiago de Cuba, Cuba), Rogelio Garcia Torrentera (Jefatura de Terapia Respiratoria, Centro de Investigación en Enfermedades Infecciosas, Instituto Nacional de Enfermedades Respiratorias (INER), Distrito Federal, Mexico), Jaime Zevallos Vásquez (Unidad de Cuidados Intensivos, Hospital Almenara, Lima, Peru), David Chiumello (Dipartimento di Anestesia, Rianimazione e Terapia del dolore, Fondazione IRCCS Cà Granda Ospedale Maggiore Policlinico, Milan, Italy), Roberto Consentini (Emergency Medicine Dept, Fondazione IRCCS Cà Granda Ospedale Maggiore Policlinico, Milan, Italy), and Zoltan Tomori (Dept of Physiology and Sleep Laboratory, Faculty of Medicine, University of Pavol Jozef Safarik, Kosice, Slovakia).

We would like to thank Mark Ryder (Istanbul American Academy, Istanbul, Turkey) for grammatical review of this article. 


\section{References}

1 International Consensus Conference in Intensive Care Medicine: noninvasive positive pressure ventilation in acute respiratory failure. Am J Respir Crit Care Med 2001; 163: 283-291.

2 Kallet R, Diaz J. The physiologic effects of noninvasive ventilation. Respir Care 2009; 54: 102-111.

3 Ambrosino N, Vagheggini G. Noninvasive positive pressure ventilation in the acute care setting: where are we? Eur Respir J 2008; 31: 874-886.

4 Levitt J, Matthay M. Treatment of acute lung injury: historical perspective and potential future therapies. Semin Respir Crit Care Med 2006; 27: 426-438.

5 Harper SA, Bradley JS, Englund JA, et al. Seasonal influenza in adults and children - diagnosis, treatment, chemoprophylaxis, and institutional outbreak management: clinical practice guidelines of the Infectious Disease Society of American. Clin Infect Dis 2009; 48: 1003-1032.

6 Kallet R. Noninvasive ventilation in acute care: controversies and acute emerging concepts. Respir Care 2009; 54: 259-263.

$7 \quad$ Hui DS, Hall SD, Chan MT, et al. Noninvasive positive-pressure ventilation: an experimental model to assess air and particle dispersion. Chest 2006; 130: 730-740.

8 Yam LY, Chan AY, Cheung TMT, et al. Noninvasive versus invasive mechanical ventilation for respiratory failure in severe acute respiratory syndrome. Chin Med J (Engl) 2005; 118: 1413-1421.

9 Fowler RA, Lapinsky SE, Hallett D, et al. Critically ill patients with severe acute respiratory syndrome. JAMA 2003; 290: 367-373.

10 WHO Interim Guidelines. Infection prevention and control of epidemic- and pandemic-prone acute respiratory diseases in health care. Geneva, World Health Organization, 2007.

11 Lin L, Xu YJ, He DP, et al. A retrospective study on clinical features of and treatment methods for 77 severe cases of SARS. Am J Chin Med 2003; 31: 821-839.

12 Cheung TM, Yam LY, So LK, et al. Effectiveness of noninvasive positive pressure ventilation in the treatment of acute respiratory failure in severe acute respiratory syndrome. Chest 2004; 126: 845-850.

13 Fowler RA, Guest CB, Lapinsky SE, et al. Transmission of severe acute respiratory syndrome during intubation and mechanical ventilation. Am J Respir Crit Care Med 2004; 169: 1198-1202.

14 Sung JJ, Wu A, Joynt GM, et al. Severe acute respiratory syndrome: report of treatment and outcome after a major outbreak. Thorax 2004; 59: 414-420.

15 Lew TW, Kwek TK, Tai D, et al. Acute respiratory distress syndrome in critically ill patients with severe acute respiratory syndrome. JAMA 2003; 290: 374-380.

16 Han F, Jiang YY, Zheng JH, et al. Noninvasive positive pressure ventilation treatment for acute respiratory failure in SARS. Sleep Breath 2004; 8: 97-106.

17 Yu IT, Xie ZH, Tsoi KK, et al. Why did outbreaks of severe acute respiratory syndrome occur in some hospital wards but not in others? Clin Infect Dis 2007; 44: 1017-1025.

18 McCracken J. Should noninvasive ventilation be considered a high-risk procedure during an epidemic? Can Med Assoc J 2009; 181: 663-664.

19 Pandemic H1N1 2009 influenza: Clinical management guidelines for adults and children. London, Department of Health, 2009. http://webarchive.nationalarchives.gov.uk/20130107105354/http://www.dh.gov.uk/en/Publicationsandstatistics/ Publications/PublicationsPolicyAndGuidance/DH_107769

20 Lum ME, McMillan AJ, Brook CW, et al. Impact of pandemic (H1N1) 2009 influenza on critical care capacity in Victoria. Med J Aust 2009; 191: 502-506.

21 Kaufman MA, Duke GJ, McGain F, et al. Life-threatening respiratory failure from H1N1 influenza 09 (human swine influenza). Med J Aust 2009; 191: 154-156.

22 Perez-Padilla R, de la Rosa-Zamboni D, Ponce de Leon S, et al. Pneumonia and respiratory failure from swineorigin influenza A (H1N1) in Mexico. N Engl J Med 2009; 361: 680-689.

23 Rello J, Rodríguez A, Ibañez P, et al. Intensive care adult patients with severe respiratory failure caused by influenza A (H1N1) in Spain. Crit Care 2009; 13: R148.

24 Kumar A, Zarychanski R, Pinto R, et al. Critically ill patients with 2009 influenza A(H1N1) infection in Canada. JAMA 2009; 302: 1872-1879.

25 Ríos FG, Estenssoro E, Villarejo F, et al. Lung function and organ dysfunctions in 178 patients requiring mechanical ventilation during the 2009 influenza A (H1N1) pandemic. Crit Care 2011; 15: R201.

$26 \mathrm{Li}$ H, Ma RC. [Clinical analysis of 75 patients with severe influenza A H1N1 in Qinghai Province]. Zhongguo Wei Zhong Bing Ji Jiu Yi Xue 2010; 22: 164-165.

27 Ramsey CD, Funk D, Miller RR, et al. Ventilator management of hypoxemic respiratory failure attributable to H1N1 novel swine origin influenza virus. Crit Care Med 2010; 38: Suppl. 4, 58-65.

28 Djibré M, Berkane N, Salengro A, et al. Non-invasive management of acute respiratory distress syndrome related to influenza A (H1N1) virus pneumonia in a pregnant woman. Intensive Care Med 2010; 36: 373-374.

29 Miller RR 3rd, Markewitz BA, Rolfs RT, et al. Clinical findings and demographic factors associated with ICU admission in Utah due to novel 2009 influenza A (H1N1) infection. Chest 2010; 137: 752-758.

30 Koegelenberg CF, Irusen EM, Cooper R, et al. High mortality from respiratory failure secondary to swine-origin influenza A (H1N1) in South Africa. QJM 2010; 103: 319-325.

31 Domínguez-Cherit G, Lapinsky SE, Macias AE, et al. Critically ill patients with 2009 influenza A(H1N1) in Mexico. JAMA 2009; 302: 1880-1887.

32 Adıgüzel N, Karakurt Z, Kalamanoğlu Balci M, et al. Influenza A (H1N1) virus pneumonia in intensive care unit. Tuberk Toraks 2010; 58: 278-285.

33 Zhang PJ, Li XL, Cao B, et al. Clinical features and risk factors for severe and critical pregnant women with 2009 pandemic H1N1 influenza infection in China. BMC Infect Dis 2012; 12: 29.

34 Conti G, Larrsson A, Nava S, et al. On the role of non-invasive (NIV) to treat patients during the H1N1 influenza pandemic. http://dev.ersnet.org/uploads/Document/63/WEB_CHEMIN_5410_1258624143.pdf Date last updated: November, 2009. Date last accessed: February 1, 2011.

35 World Health Organization. Avian Influenza, including influenza A (H5N1), in humans: WHO interim infection control guidelines for health care facilities. Geneva, World Health Organization, 2007. 
Winck JC, Marinho A. Non-invasive ventilation in acute respiratory failure related to 2009 pandemic Influenza A/H1N1 virus infection. Crit Care 2010; 14: 408.

37 Esquinas A. International survey acute respiratory failure in H1N1 infection. Am J Respir Crit Care Med 2010; 181 : A6117.

38 Hajjar LA, Mauad T, Galas FR, et al. Severe novel influenza A (H1N1) infection in cancer patients. Ann Oncol 2010; 21: 2333-2341.

39 Nin N, Soto L, Hurtado J, et al. Clinical characteristics and outcomes of patients with 2009 influenza A (H1N1) virus infection with respiratory failure requiring mechanical ventilation. J Crit Care 2011; 26: 186-192.

40 Louriz M, Mahraoui C, Azzouzi A, et al. Clinical features of the initial cases of 2009 pandemic influenza A (H1N1) virus infection in a university hospital of Morocco. Int Arch Med 2010; 3: 26.

41 Adlhoch C, Wadl M, Behnke M, et al. Pandemic influenza A(H1)pdm09 in hospitals and intensive care units results from a new hospital surveillance, Germany 2009/2010. Influenza Other Respir Viruses 2012; 6: 162-168.

42 Hui DS, Lee N, Chan PK. Clinical management of pandemic 2009 influenza A (H1N1) infection. Chest 2010; 137: 916-925.

43 Liu L, Zhang RF, Lu HZ, et al. Sixty-two severe and critical patients with 2009 influenza A (H1N1) in Shanghai, China. Chin Med J (Engl) 2011; 124: 1662-1666.

44 Martin-Loeches I, Diaz E, Vidaur L, et al. Pandemic and post-pandemic influenza A (H1N1) infection in critically ill patients. Crit Care 2011; 15: R286.

45 Grasselli G, Bombino M, Patroniti N, et al. Management of acute respiratory complications from influenza A (H1N1) infection: experience of a tertiary-level intensive care unit. Minerva Anestesiol 2011; 77: 884-891.

46 Timenetsky KT, Aquino SH, Saghabi C, et al. High success and low mortality rates with non-invasive ventilation in influenza A H1N1 patients in a tertiary hospital. BMC Res Notes 2011; 4: 375.

47 Belenguer-Muncharaz A, Reig-Valero R, Altaba-Tena S, et al. Noninvasive mechanical ventilation in severe pneumonia due to H1N1 virus. Med Intensiva 2011; 35: 470-477.

48 Masclans JR, Pérez M, Almirall J, et al. Early non-invasive ventilation treatment for severe influenza pneumonia. Clin Microbiol Infect 2013; 19: 249-256.

49 Jensen PA, Lambert LA, Iademarco MF, et al. Guidelines for preventing the transmission of Mycobacterium tuberculosis in health-care settings, 2005. MMWR Recomm Rep 2005; 54: 1-141.

50 Sekino $\mathrm{H}$, Ohi M, Chin K, et al. [Long-term artificial ventilation by nasal intermittent positive pressure ventilation; 6 cases of domiciliary assisted ventilation]. Nihon Kyobu Shikkan Gakkai Zasshi 1993; 31: 1377-1384.

51 Tsuboi T, Ohi M, Chin K, et al. [Outcome of long-term nasal intermittent positive pressure ventilation in 41 patients with hypercapnic respiratory failure]. Nihon Kyobu Shikkan Gakkai Zasshi 1996; 34: 959-967.

52 Kondoh Y, Taniguchi H, Ikuta N, et al. [Outcomes of noninvasive positive-pressure ventilation in patients with acute hypercapnia complicating chronic respiratory failure]. Nihon Kyobu Shikkan Gakkai Zasshi 1997; 35: 960-964.

53 Machida K, Tanaka T, Tsuboi T, et al. [Report of national survey on death due to tuberculosis in 1994 in national hospitals and the treatment and prognosis of tuberculosis patients with mechanical ventilation]. Kekkaku 1998; 73: 739-745.

54 Schulz MR, Herth FJ, Wiebel M, et al. Intermittent positive pressure ventilation in post tuberculosis syndrome. Pneumologie 1999; 53: Suppl. 2, S116-S119

55 Machida K. [Management of respiratory failure in patients with pulmonary tuberculosis]. Kekkaku 2003; 78: 101-105.

56 Agarwal R, Gupta D, Handa A, et al. Noninvasive ventilation in ARDS caused by Mycobacterium tuberculosis: report of three cases and review of literature. Intensive Care Med 2005; 31: 1723-1724.

57 Utsugi M, Ishizuka T, Hisada T, et al. Acute respiratory failure associated with miliary tuberculosis successfully treated with sivelestat sodium hydrate. Intern Med 2006; 45: 1069-1070.

58 Aso H, Kondoh Y, Taniguchi H, et al. Noninvasive ventilation in patients with acute exacerbation of pulmonary tuberculosis sequelae. Intern Med 2010; 49: 2077-2083.

59 Chin K, Ohi M, Kuno K. [Noninvasive ventilatory support on chronic respiratory failure with hypoventilation]. Bull Chest Dis Res Inst Kyoto Univ 1989; 22: 50-57.

60 Prats Soro E, Escarrabill Sanglas J, Farrero Muñoz E, et al. Ventilacion mecanica a domicilo como tratamiento de las secuelas de la cirugia de la tuberculosis [Home mechanical ventilation as a treatment of the sequelae of tuberculosis surgery]. An Med Interna 1999; 16: 35-37.

61 Guerin C, Girad R, Chemorin C, et al. Facial mask noninvasive mechanical ventilation reduces the incidence of nosocomial pneumonia. Intensive Care Med 1997; 23: 1024-1032.

62 Hui DS, Chow BK, Ng SS, et al. Exhaled air dispersion distances during noninvasive ventilation via different respironics face masks. Chest 2009; 136: 998-1005.

63 American Association for Respiratory Care. AARC SARS page. www.aarc.org/resources/sars/index.asp Date last accessed: December 4, 2008.

64 Antonelli M, Conti G, Esquinas A, et al. A multiple-center survey on the use in clinical practice of noninvasive ventilation as a first-line intervention for acute respiratory distress syndrome. Crit Care Med 2007; 35: 18-25.

65 Centers for Disease Control and Prevention. Interim recommendations for infection control in health-care facilities caring for patients with known or suspected avian influenza. www.cdc.gov/flu/avian/professional/infect-control.htm Date last updated: May 21, 2004. Date last accessed: September 18, 2012. 